\title{
DAMAGE FORMATION AND CALCULATION OF ENERGY LOSS DURING IMPLANTATION OF ANTIMONY AND BORON ION IN SILICON TARGET
}

G. P. Adhikari and H. K. Limbu

Journal of Nepal Physical Society

Volume 5, Issue 1, October 2019

ISSN: $2392-473 X$

Editors:

Dr. Vinaya Kumar Jha

Dr. Binod Adhikari

Dr. Kapil Adhikari

JNPS, 5 (1), 103-110 (2019)

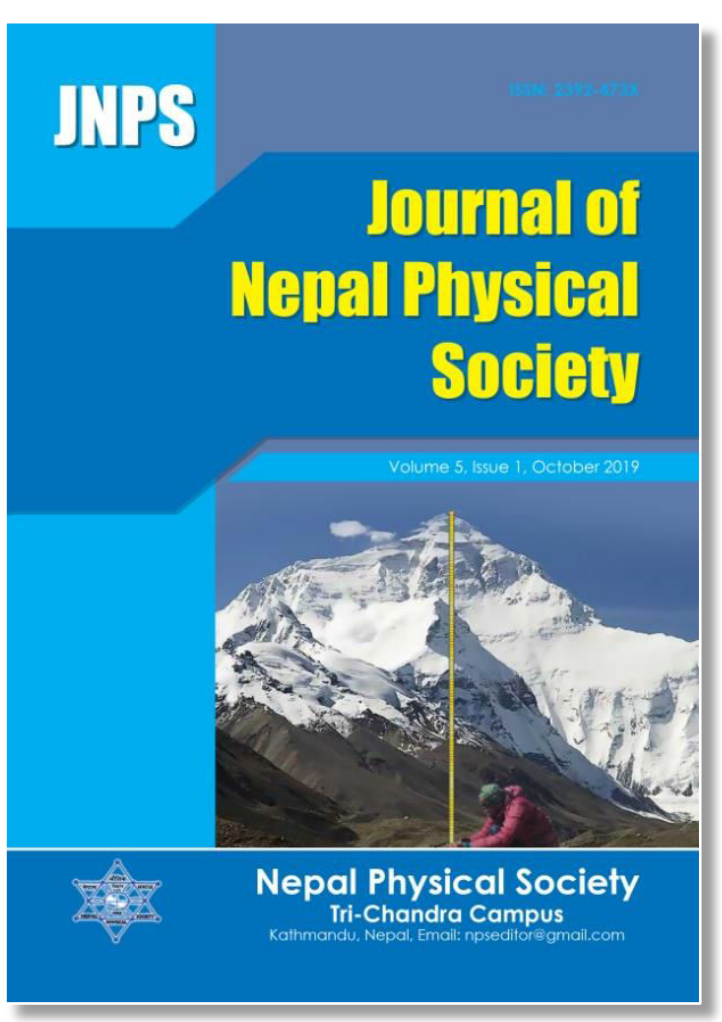

Published by:

Nepal Physical Society

P.O. Box: 2934

Tri-Chandra Campus

Kathmandu, Nepal

Email:npseditor@gmail.com 


\title{
DAMAGE FORMATION AND CALCULATION OF ENERGY LOSS DURING IMPLANTATION OF ANTIMONY AND BORON ION IN SILICON TARGET
}

\author{
G. P. Adhikari* and H. K. Limbu \\ Department of Physics, Tri-Chandra Multiple Campus, Tribhuvan University, Kathmandu \\ "Corresponding Email: gpadhikari67@gmail.com
}

\begin{abstract}
The aim of this research work is a computational study of damage profile and visualization of the ion implantation graph obtained from the simulation method using SRIM-2013 software. SRIM (Stopping and Range of Ions in Matter) helps in calculating the energy required for an ion to obtain maximum concentration for desirable range and TRIM (Transport of Ions in Matter) is used to calculate doping statistics and calculation of energy loss. The main objective of this work is to get knowledge about the graphical study of ion range, distribution, stopping power and energy loss during implantation of antimony and boron ion on the silicon target within 0 to $3500 \AA$ target depth. The implantations of 10,000 antimony and boron ions are accelerated by 350 $\mathrm{keV}$ and $45 \mathrm{keV}$ energy in silicon monolayer target to obtain maximum defects concentration. The result of the ionization process indicates that $11.68 \%$ of the total energy of antimony ion and $65.25 \%$ of the total energy of boron ion are lost during the ionization process. This indicates that lighter boron ion causes more ionization than the heavier antimony ion for the same projected range.
\end{abstract}

Keywords: Ion implantation, Semiconductor, Doping statistics, Stopping power, Ion range, Defect concentration.

\section{INTRODUCTION}

\section{Ion Implantation}

During ion implantation, the energetic impurity ions penetrate the target and finally combine with the target, create holes or free electrons. These holes and electrons are responsible for conductivity in semiconductor devices [1]. During the ion target collision process, the impurity ion suffers multiple collisions with the nucleus as well as electrons. Ultimately the accelerated ion loses all its energy and rests in the target atoms, creating additional charge careers (holes and electrons) [2].

Ion implantation is a doping method widely used in semiconductor technology for the fabrication of several bipolar devices in silicon substrate. This technique helps to inject any element into the nearsurface region of substrate with good accuracy. Antimony is a dopant suitable for the fabrication of n-type junction; this is due to its high mass and low diffusion in silicon [3-5].

\section{Silicon as a substrate for ion implantation}

Addition of an element such as boron atom which can be substituted for a silicon atom in the crystal structure which provides one less valance electron than silicon loses an electron to it. The positive holes created by the shift in electron allow extrinsic semi conduction of p-type refer to as positive. Addition of an element such as antimony atom which can also be substituted for a silicon atom in the crystal which provides an extra valance electron of it's within the lattice. These electrons allow semiconductor of the ntype refer to as negative $[4,6]$.

\section{Selections of implanting ions}

We are interested in the investigation of damage profiles of silicon at the range of $1550 \AA$ when doped with p-type ions and n-type ions. We choose the element, boron, of which have comparatively less masse as compare to silicon and act as p-type dopants ion and antimony, is taken as n-type dopants ion which acts as a donor impurity and have comparable high mass then silicon[1,7].

\section{Objectives}

The overall objective of this research work is to study the effects on silicon when p-type, boron, and n-type, antimony dopants are implanted. 
Some specific objectives are:

- To identify the energy needed to implant $\mathrm{Sb}$ ions and $\mathrm{B}$ ion in a silicon at the estimated range of $1550 \AA$.

- To calculate the various energy losses to the target electrons (electronic losses) and to the target nuclei (phonons).

- To study the various effect of silicon single layer creating displacements, vacancies, and replacement collision.

\section{MATERIALS AND METHODS}

\section{Simulation Methodology}

This simulation software for the ion implantation is SRIM (stopping and range of ions in matter). The software was developed by Ziegler and first published in 1985. SRIM is a group of computer programs that calculate the interaction of ions with matter. The core of SRIM is a program transport of ions in matter (TRIM). SRIM calculates the interaction of energetic ions with amorphous targets and presents those interactions statistically and graphically which make the implantation process easier to understand [8].

\section{Theoretical Methodology}

Ion implantation is used to put specific amounts of n-type and p-type dopants into a semiconductor.

\section{Stopping Power}

The stopping power is an essential parameter when ions penetrate matter. It defines how much energy per unit path length an ion loses when penetrating a crystal, thus the stopping power is usually measured in units of eV/Å. When energetic ions are allowed to penetrate through the target surface, a series of screened Coulomb collision occurs where the ion's energy gets divided into two stopping parameters, one is nuclear stopping and another is electronic stopping. Nuclear stopping is the energy loss by ion to the target nuclei per unit length of ion. It is an elastic collision between two atoms and can be described by classical kinematics. Electronic stopping is the energy loss by ion to the electrons of target atoms per unit path. It is an inelastic collision and the theoretical model is quite complex $[1,9,10-12]$.

\section{Range Distribution}

An energetic ion entering a target will, through with the target nuclei and electrons lose its energy and finally come to rest. The range distribution will depend primarily on the energy, mass, and atomic number of the incoming ions, the mass and atomic number of target atoms, the density of the target. The total distance that the ion travels in coming to rest is called its range and the projection of this distance onto the direction of incidence is called the projected range. There should be a distribution of stopping points, or a range distribution, which are characterized by quoting a mean ion range of the distribution function like skewness and kurtosis [5, 10-12]

\section{Collision Events}

Total target displacements are the number of atoms knocked off their target lattice site. A vacancy is the hole left behind when a recoil atom moves from its original site. If a moving atom strikes a stationary target atom and transfers more than its displacement energy to it, and the initial atom, after the collision, does not have enough energy to move onwards, and it is the same element as the atom it struck, then it just replaces that atom in the target and there is no vacancy created. These are termed as replacement collisions.

Displacements $=$ Vacancies + Replacement Collisions

If a cascade atom leaves the target volume, it is no longer followed. TRIM will follow atoms indefinitely as they go sideways, even though they leave your screen. But if they go through either target surface they are discarded and not counted. So vacancies occur within the target, and the final resting place of a moving recoil atom can be some distance from its vacancy [10-12].

\section{RESULT AND DISCUSSION}

\section{Energy Calculations using SRIM}

When implanting antimony and boron ions in silicon target, determination of the energy of respective impurities ion is required. Using Stopping Range Table of SRIM calculation, energy range to implant antimony and boron ion in silicon monolayer target is obtain below.

Antimony in Silicon : Ion = Antimony, Atomic number $=51$, Atomic mass $=121.76 \mathrm{amu}$

Target Composition: Atomic name $=$ Silicon, Atomic number $=14$, Atomic mass $=28.0855 \mathrm{amu}$, Target density $=2.328 \mathrm{~g} / \mathrm{cm}^{3}=$ $4.9770 \times 10^{22}$ atoms $/ \mathrm{cm}^{3}$, Stopping units $=\mathrm{eV} / \AA$

Boron in Silicon: Ion $=$ Boron, Atomic number $=$ 5, Atomic mass $=10.81 \mathrm{amu}$ 
Table 1: Energy calculation for Antimony ion.

\begin{tabular}{|c|c|c|c|}
\hline $\begin{array}{c}\text { Ion Energy } \\
(\mathbf{K e V})\end{array}$ & $\begin{array}{c}\mathbf{d E} / \mathbf{d x} \\
\text { Electrical }(\mathbf{e V} / \mathbf{\AA})\end{array}$ & $\begin{array}{c}\mathbf{d E} / \mathbf{d x} \\
\text { Nuclear }(\mathbf{e V} / \mathbf{\AA})\end{array}$ & $\begin{array}{c}\text { Projected Range } \\
(\mathbf{\AA})\end{array}$ \\
\hline 200 & 1.08 & 8.58 & 944 \\
\hline 225 & 1.15 & 8.54 & 1044 \\
\hline 250 & 1.28 & 8.48 & 1144 \\
\hline 275 & 1.50 & 8.42 & 1243 \\
\hline 300 & 1.65 & 8.34 & 1341 \\
\hline 325 & 1.75 & 8.27 & 1439 \\
\hline $\mathbf{3 5 0}$ & $\mathbf{1 . 8 3}$ & $\mathbf{8 . 1 9}$ & $\mathbf{1 5 3 7}$ \\
\hline 375 & 1.89 & 8.10 & 1635 \\
\hline 400 & 1.93 & 8.02 & 1733 \\
\hline 450 & 2.00 & 7.86 & 1933 \\
\hline 500 & 2.07 & 7.69 & 2134 \\
\hline 550 & 2.14 & 7.53 & 2338 \\
\hline 600 & 2.21 & 7.38 & 2544 \\
\hline
\end{tabular}

The objective of this work was to study the effect profiles and energy losses with maximum defects concentration at $1550 \AA$ A. Energy calculation by SRIM in table 1, demonstrate that $350 \mathrm{keV}$ energy is required for antimony to have the projected range of $1537 \AA$ in silicon which is close enough to estimate range, So I implant 10,000 antimony ions accelerated by $350 \mathrm{keV}$ energy in silicon monolayer target in order to obtain maximum defects concentration.

Table 2: Energy calculation for Boron ion.

\begin{tabular}{|c|c|c|c|}
\hline $\begin{array}{c}\text { Ion Energy } \\
(\mathbf{K e V})\end{array}$ & $\begin{array}{c}\mathbf{d E} / \mathbf{d x} \\
\text { Electrical(eV/ } \mathbf{A})\end{array}$ & $\begin{array}{c}\mathbf{d E} / \mathbf{d x} \\
\text { Nuclear(eV/ } \mathbf{\AA})\end{array}$ & $\begin{array}{c}\text { Projected } \\
\text { Range }(\mathbf{\AA})\end{array}$ \\
\hline 27.5 & 6.96 & 2.49 & 1038 \\
\hline 30 & 7.42 & 2.40 & 1122 \\
\hline 32.5 & 7.83 & 2.32 & 1204 \\
\hline 35 & 8.21 & 2.24 & 1284 \\
\hline 37.5 & 8.55 & 2.17 & 1364 \\
\hline 40 & 8.86 & 2.11 & 1442 \\
\hline $\mathbf{4 5}$ & $\mathbf{9 . 4 1}$ & $\mathbf{1 . 9 9}$ & $\mathbf{1 5 9 5}$ \\
\hline 50 & 9.87 & 1.89 & 1746 \\
\hline 55 & 1.03 & 1.79 & 1895 \\
\hline 60 & 1.06 & 1.71 & 2042 \\
\hline 65 & 1.09 & 1.64 & 2188 \\
\hline 70 & 1.12 & 1.57 & 2618 \\
\hline 80 & 1.17 & 1.46 & \\
\hline
\end{tabular}



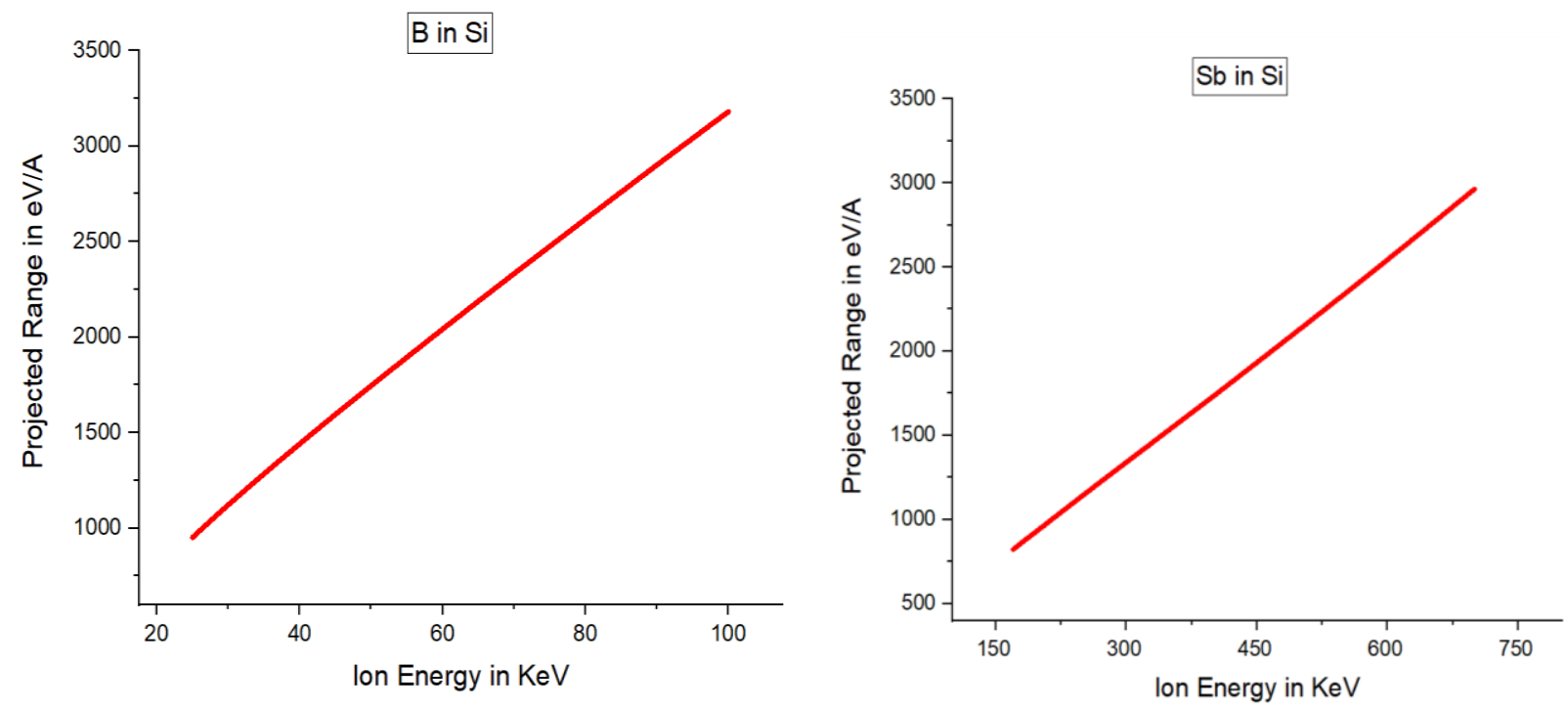

Fig.1: The graph between ion energy and projected range for $S b$ and $B$ ions.

Energy calculation by SRIM in table 2, the exact energy required for p-type boron dopants to obtain maximum defects at $1595 \AA$ is $45 \mathrm{keV}$. Therefore, I can now implant 10,000 boron ions that are accelerated by $45 \mathrm{keV}$ energy in silicon monolayer target to obtain maximum defects concentrations.

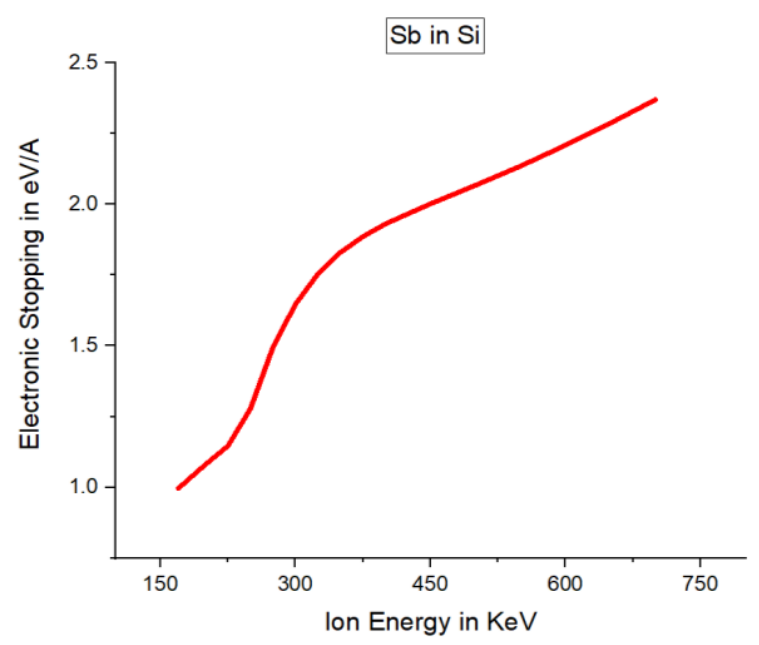

\section{Stopping Power}

The stopping power of the target is defined by the energy loss (E) per unit path length $(x)$ of the ion. The energy loss of ions is basically separated in to two distinct processes as electronic and nuclear stopping power.

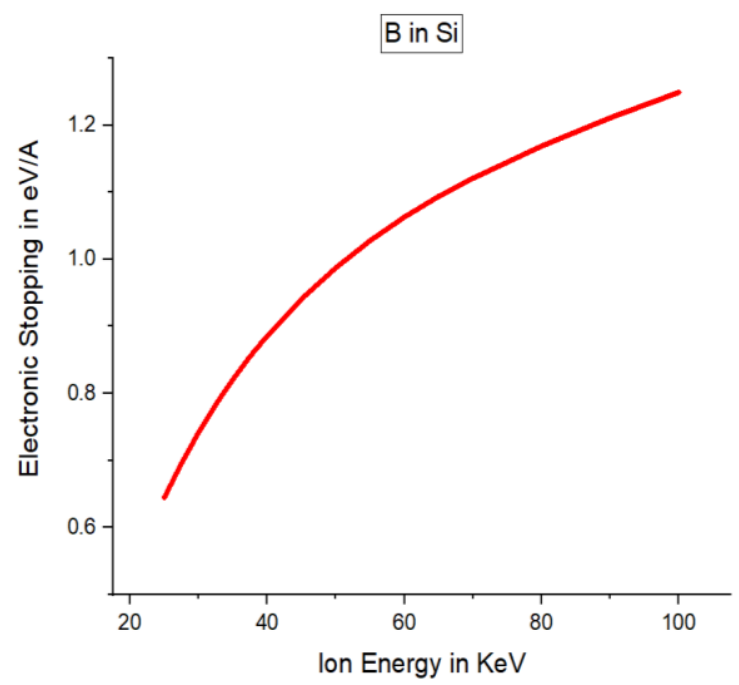

Fig.2: Graph between Ion Energy (KeV) and Electronic Stopping (eV/ $)$ during Implantation of $\mathrm{Sb}$ and $\mathrm{B}$ ions in Silicon target.

Electronic stopping is caused by the interaction between the incoming ion and the electron in the target when the ion is moving through it. For higher energetic implanted ions, the electronic stopping is major energy loss due to inelastic collision with the electron cloud. As boron has less mass, for the same range of ion energy, it acquires higher velocity than that of antimony ion of the same range of ion energy. Since electronic stopping is proportional to the ion velocity, ion with less velocity has less electronic stopping. 

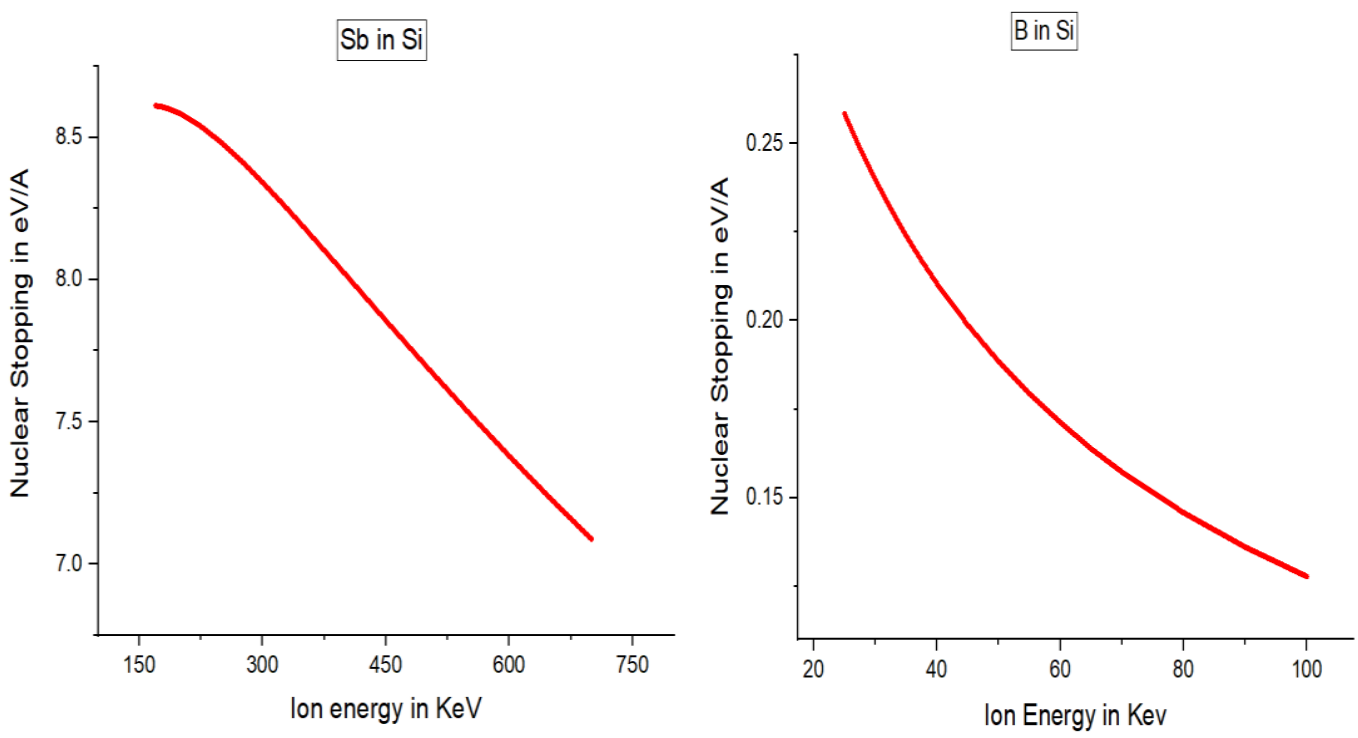

Fig. 3: Graph between ion energy and nuclear stopping for Sb and B ions in silicon target.

Nuclear stopping is caused by the collision between two atoms. Nuclear stopping is elastic, so energy lost by the incoming ion is transferred to the target atom. As ion energy is increased the ion velocity will increase cause of to decrease in nuclear stopping. When ion energy is higher up to few Mev during implantation of ions, Electronic stopping power dominates nuclear stopping power and hence the conductivity of the semiconductor can be increased.

\section{TRIM calculation for Antimony- Silicon and Boron- Silicon Collision}

This section deals with the detail discussions of outcomes obtained from TRIM. Various distribution curves of range, concentration versus depth and energy loss per ions versus depth are the graphical results that are interpreted in this section. The amount of energy losses to ionization, phonon, and vacancy production are the major outcomes here.

\section{Energy Loss during Ionization}

The energy loss - target depth curves for ionization of silicon atoms by the incident antimony and boron energetic ions are shown in figures. The area under the curve calculations shows that antimony ions loss $40.88 \mathrm{keV}(11.68 \%)$ of their energy in the ionization process whereas that for boron ion is $29.36 \mathrm{keV}(65.25 \%)$. The energy consumed by the ionization of silicon recoils resulting from $\mathrm{Sb}$ ion amounts to $108.01 \mathrm{keV}(30.86 \%)$ while that of B recoils gives $3.40 \mathrm{k} \mathrm{eV}(7.56 \%)$.

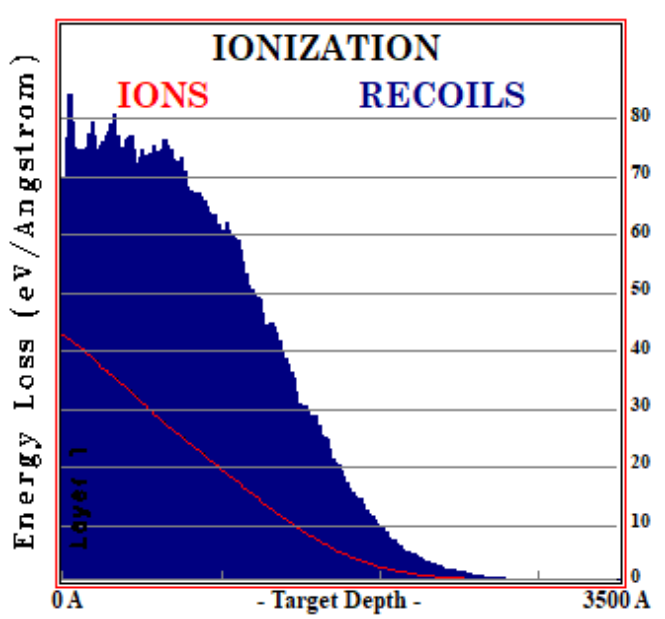

Fig.4: Ions and recoils ionization curves for Sb ions.

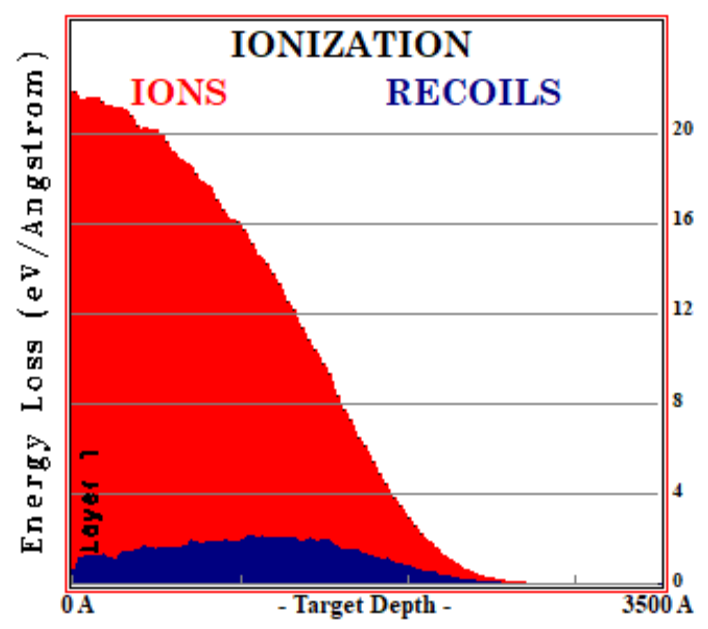

Fig. 5: Ions and recoils ionization curves for B ions. 
The result of the ionization process indicates that $11.68 \%$ of the total energy of Sb ion and $65.25 \%$ of the total energy of $\mathrm{B}$ ion are lost during ionization process. This indicates that lighter $\mathrm{B}$ ion causes more ionization than the heavier $\mathrm{Sb}$ ion for the same projected range.

\section{Ion Ranges}

The nature of ion range during doping of antimony and boron ion on silicon target with the energy of $350 \mathrm{KeV}$ and $45 \mathrm{KeV}$ respectively within the target depth of $3500 \AA$, is shown in the figure below. In this doping process of $\mathrm{Sb}$ ion in Silicon target ion rang of $1583 \AA$ is achieved with straggle of $455 \AA$.

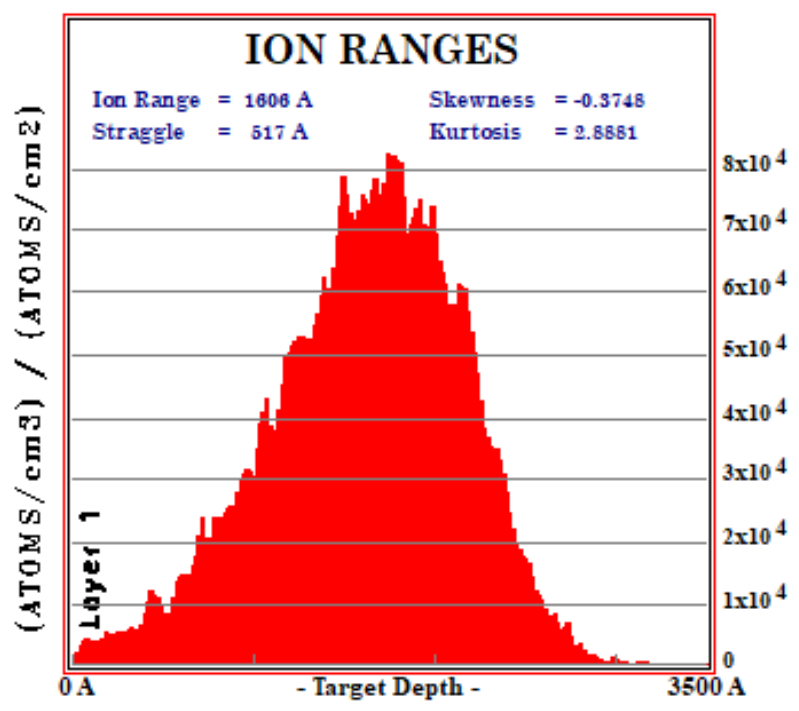

Fig. 6: Ion range distribution curves for Sb ions.

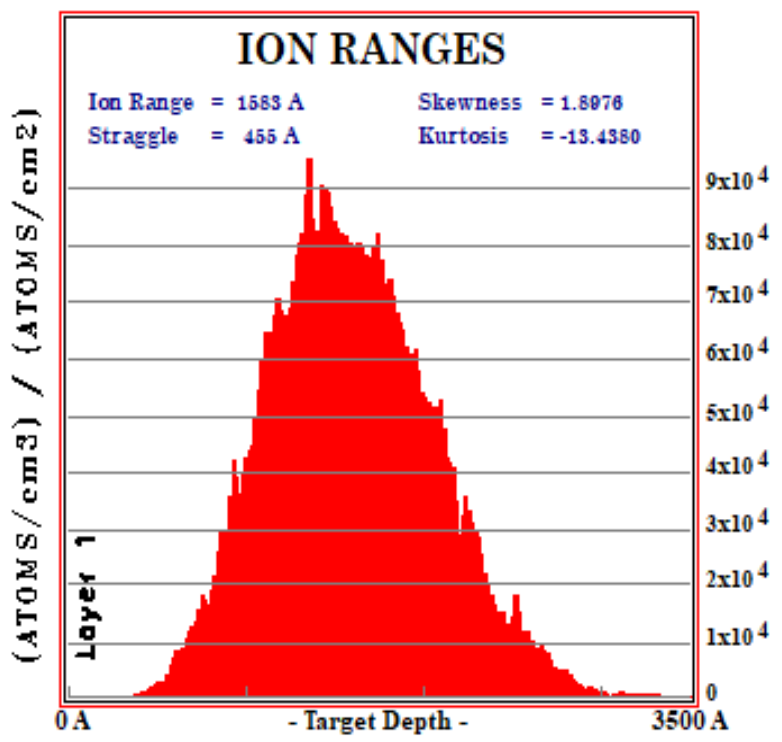

Fig. 7: Ion range distribution curves for B ions.
The skewness for the curve is calculated to be 1.8976 (positive coefficient) which suggest that right tail of the distribution is longer the left so the graph is skewed right. The kurtosis of the curve is calculated -13.4380 (Negative kurtosis) which indicates that the distribution has lighter tails and a flatter peak then the normal distribution.

The nature of ion range during this doping process for $45 \mathrm{keV}$ accelerated $\mathrm{B}$ ion in Silicon target. In this doping process, ion rang of $1606 \AA$ is achieved with straggle of $517 \AA$. The skewness for the curve is calculated to be -0.3748 (negative coefficient) which suggests that the left tail of the distribution is longer the right so the graph is skewed left. The kurtosis of the curve is calculated 2.8881 (Positive kurtosis) which indicates that the distribution has heavier tails than the normal distribution.

Table 3: Showing mean range values of $\mathrm{Sb}$ and $\mathrm{B}$ ion in Silicon target.

\begin{tabular}{|l|l|l|}
\hline Parameter & Antimony & Boron \\
\hline Ion Range & 1583 & 1606 \\
\hline Straggle & 455 & 517 \\
\hline Skewness & 1.8976 & -0.3748 \\
\hline Kurtosis & -13.4380 & 2.8881 \\
\hline
\end{tabular}

\section{Collision events}

The results of the collision events of Sb and B ions give the total target displacement with Silicon target as shown in figure. The curve shows the total target displacements. This is the number of atoms knocked off their target lattice site.

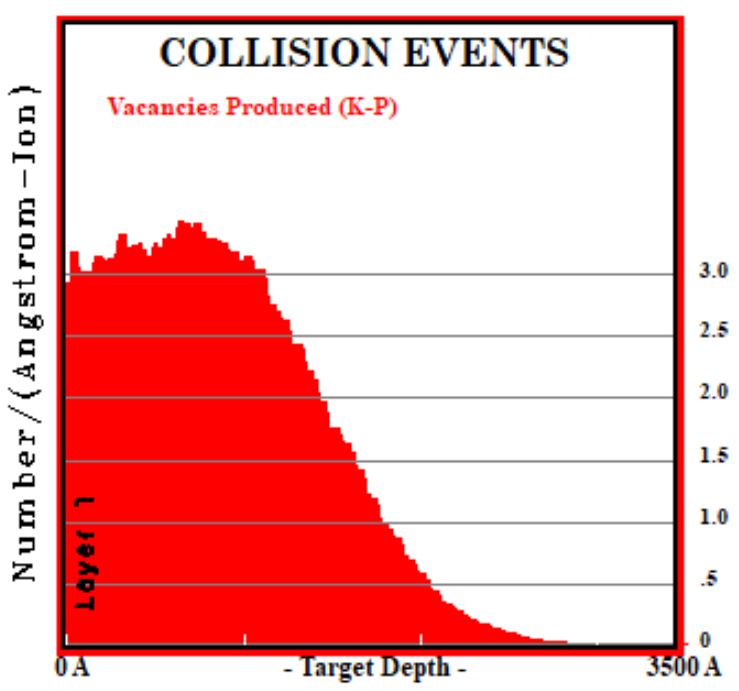

Fig. 8: Silicon vacancy distribution for Sb ions. 


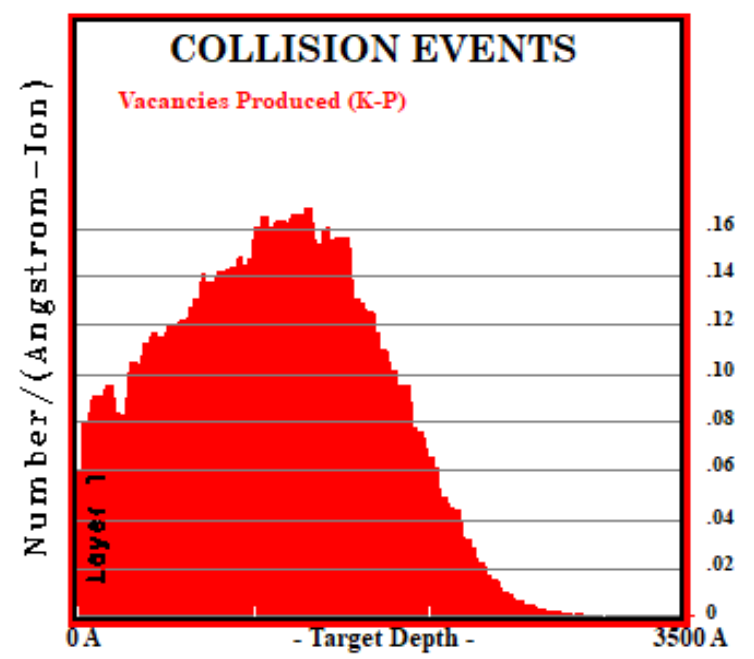

Fig. 9: Silicon vacancy distribution for B ions.

The area under the curve calculations gives values for total displacements of 5351/ ions and 316/ ions for antimony and boron ion implantation on the silicon target. Target vacancy production amounts to $0.10 \%$ and $0.23 \%$ of total ion energy for $\mathrm{Sb}$ and $\mathrm{B}$ whereas for Si recoils atoms vacancy production accounts to $2.95 \%$ and $1.16 \%$ of total energy respectively.

A summary of the collision event is shown in the table below

Table 4: Defect produced during ion implantation of $\mathrm{Sb}$ and B ion in Silicon target

\begin{tabular}{|l|l|l|}
\hline & Arsenic & Boron \\
\hline Total displacement/ ion & 5351 & 316 \\
\hline Total vacancy /ion & 5351 & 316 \\
\hline Replacement Collision/ion & 0 & 0 \\
\hline
\end{tabular}

During the study of collision events, we know that Displacement $=$ Vacancies + Replacement Collision

\section{Energy loss to phonon}

The results show that the contribution of $\mathrm{Sb}$ and $\mathrm{B}$ ions in phonon production is extremely small as compared with that produce by recoils. The energy loss by ions and recoils for Sb ions implantation is $0.21 \%$ and $54.20 \%$ of total energy $(350 \mathrm{keV})$ while that loss from B ions is $0.81 \%$ and $24.99 \%$ of total energy (45 keV).

\section{Lateral Range Distribution}

This plot summarizes the lateral and radial spread of ions within the target. The lateral projected range is defined as the average of the values of the projected displacements from the $\mathrm{x}$ - axis. Where as radial range is the mean radial displacement from the $\mathrm{x}$-axis. The ion state section of TRIM calculation is presented below.

Table 5: Longitudinal, Radial and Lateral Projected range for $\mathrm{Sb}$ and $\mathrm{B}$ ions.

\begin{tabular}{|l|c|c|}
\hline & $\begin{array}{c}\text { Range of } \\
\text { Sb (A) }\end{array}$ & $\begin{array}{c}\text { Range of B } \\
(\mathbf{\AA})\end{array}$ \\
\hline Longitudinal & 1583 & 1606 \\
\hline $\begin{array}{l}\text { Lateral } \\
\text { Projection }\end{array}$ & 255 & 471 \\
\hline Radial & 400 & 739 \\
\hline
\end{tabular}

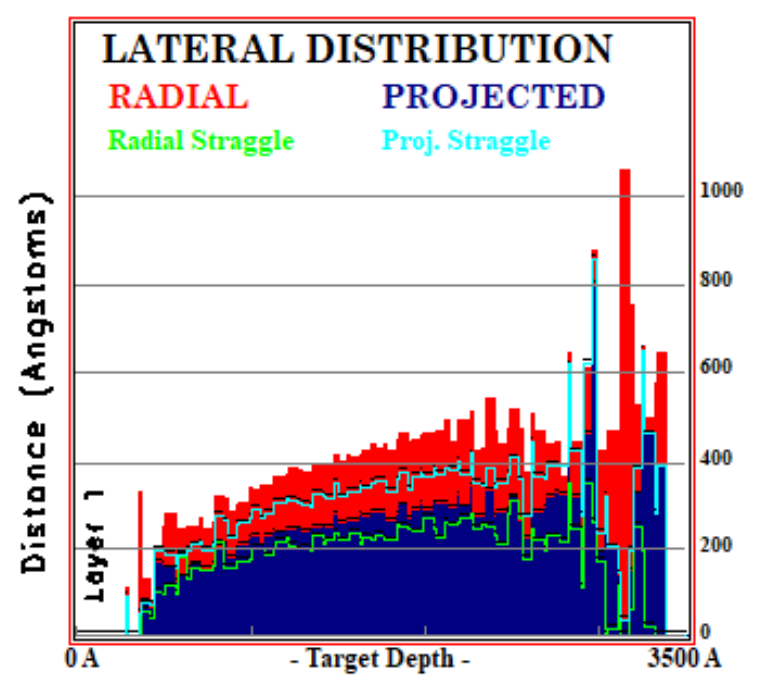

Fig.10: Lateral distribution curve for Sb ions.

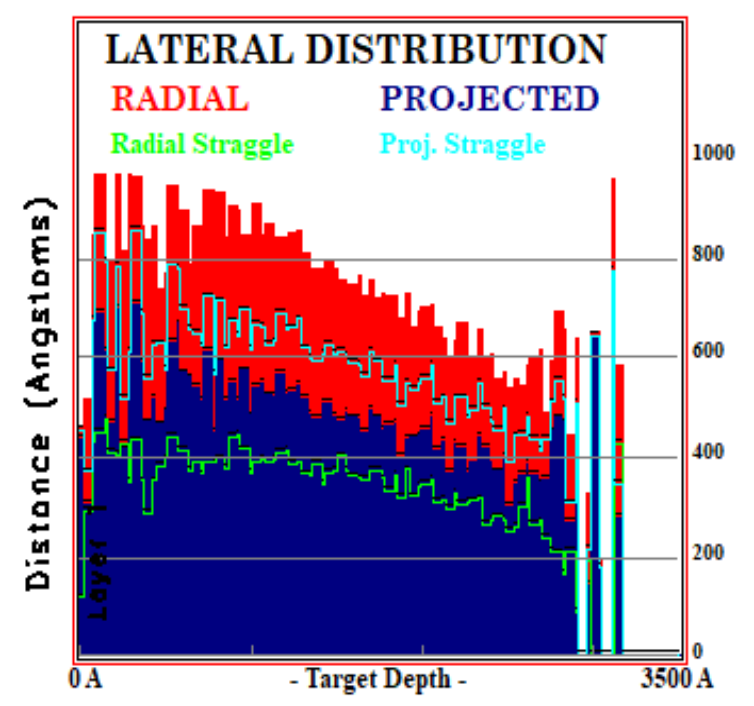

Fig.11: Lateral distribution curve for B ions. 
Table 6: Longitudinal, Radial and Lateral Projected range for $\mathrm{Sb}$ and $\mathrm{B}$ ions.

\begin{tabular}{|c|c|c|}
\hline & $\begin{array}{c}\text { Straggle for } \\
\text { Sb (A) }\end{array}$ & $\begin{array}{c}\text { Straggle for } \\
\text { B (A) }\end{array}$ \\
\hline Longitudinal & 455 & 517 \\
\hline $\begin{array}{l}\text { Lateral } \\
\text { Projection }\end{array}$ & 326 & 584 \\
\hline Radial & 226 & 386 \\
\hline
\end{tabular}

Comparatively, the lateral projected range and struggle, radial range and struggle for $\mathrm{B}$ ion are high at the surface and gets decrease gradually with the increase in target depth. But that for Sb ions is low at the surface and increases gradually with the increase in target depth.

\section{CONCLUSIONS}

Ion implantation of $\mathrm{Sb}$ and $\mathrm{B}$ ion in silicon target shows that most of the energy loss is due to ionization and phonon production due to ion and due to recoils of ion by the target atom. Sb ions loss $40.88 \mathrm{keV}(11.68 \%)$ of their energy in the ionization process whereas that for B ions is 29.36 $\mathrm{keV}(65.25 \%)$. The energy consumed by the ionization of silicon recoils resulting from $\mathrm{Sb}$ ions amounts to $108.01 \mathrm{keV}(30.86 \%)$ while that of B ions recoils gives $3.40 \mathrm{keV}(7.56 \%)$. Total displacements of 5351/ ions and 316/ ions for $\mathrm{Sb}$ and B ion implantation on the Silicon target. Target vacancy production amounts to $0.10 \%$ and $0.23 \%$ of total ions energy for $\mathrm{Sb}$ and $\mathrm{B}$ whereas for $\mathrm{Si}$ recoils atoms vacancy production accounts to $2.95 \%$ and $1.16 \%$ of total energy respectively. The results show that the contribution of $\mathrm{Sb}$ and $\mathrm{B}$ ions in phonon production is extremely small as compared with that produced by recoils. The energy loss by ions and recoils for $\mathrm{Sb}$ ions implantation is $0.21 \%$ and $54.20 \%$ of total energy $(350 \mathrm{keV})$ while that loss from B ions is $0.81 \%$ and $24.99 \%$ of total energy $(45 \mathrm{keV})$. These statistics can be used for the further study of semiconductor devices.

\section{REFERENCES}

[1] Nasti, M.; Mayer J.; Hirvonen; J.K. Ion-solid interactions: fundamentals and applications, Cambridge University Press (1996).

[2] Tyagi, M.S. Introduction to semiconductor materials and devices, John Wiley \& Sons (2008).

[3] Valbusa, U.; Boragno, C.; Mongeto, F.B. Nanostructuring surfaces by ion sputtering, Journal of Physics Condensed Matter, 14: 81538175 (2002). DOI: 10.1142/S179362811000616.

[4] Serrar, H.; Labbani, R.; Benazzouz, C. Experimental characterization of antimony dopant in silicon substrate, (2016), Acta Physica Polonica A, 130: 51-54 (2016). https://www. researchgate.net/publication/307612826.

[5] Lawrence, A. L; Justin, M. W. Ion implantation of semiconductor doping and material modification, Reviews of Accelerator Science and Technology, 4: 11-40 (2011). https://www. researchgate.net/publication/263990177.

[6] Willams, J. S. Ion implantation in semiconductors, Material Science and Engineering A, 253:8-15 (1998).

[7] Kabadayi, O. The channeled stopping powers of boron ions and range calculation in $\mathrm{Si}$, Czechoslovak Journal of Physics, vol. 54: 461472 (2004). https://www.researchgate.net/ publication/258937256.

[8] Ziegler, J.F; Ziegler,M.D; Bierseck, J.P. SRIMThe stopping range of ion in matter, Nuclear Instruments and Methods in Physics Research B, vol. 268: 1818-1823 (2010).

[9] Ebrahiem, S.A.; Hady, F.M.; Jassim, M.K.; Tawfeek, H.M. Simulation study of sputtering yield of $\mathrm{Zn}$ target bombarded by xenon ions, $I b n$ Al- Haitham J. for Pure \& Appl. Sci, 29(2): 104114 (2016). https://www.researchgate.net/ publication/325533884.

[10] Kinchin, Pease R.S. Rep. Prog. Phys., 18, 1 (1955)

[11] Norgett, M.J; Robinson, M.T; Torrens, I.M. Nucl. Eng. Design, 33, 50 (1974).

[12] Sigmund P. Radiation Effect, 1, 15 (1969). 